\title{
Gamma knife radiosurgery (GKRS) for pineal region tumors: a study of 147 cases

\author{
Wentao $\mathrm{Li}^{1+}$, Binfei Zhang ${ }^{2}$, Wenxing Kang ${ }^{3 \dagger}$, Boning Dong ${ }^{4 \dagger}$, Xudong Ma $^{1}$, Jinning Song ${ }^{1 *}$, Yonghong Liu ${ }^{5}$ \\ and Zhenqiang Liang ${ }^{6}$
}

\begin{abstract}
Background: The purpose of this study is to evaluate the effectiveness of gamma knife radiosurgery (GKRS) in the treatment of pineal region tumors (PRTs).

Methods: We retrospectively reviewed 147 cases of PRTs primarily treated with GKRS at our hospital between 1999 and 2009. Mean follow-up time was 67 months (range 60.5-100.1). The local tumor control rates (LTCRs) and overall survival rates were calculated to evaluate the results of the GKRS treatment.

Results: At 2 months after GKRS, tumor volume was significantly reduced in 91 cases (61.9\%). At 6 months, average tumor volume was $4.2 \mathrm{~cm}^{3}$ as compared to $8.47 \mathrm{~cm}^{3}$ before GKRS. By 1 year after GKRS, the tumor completely disappeared in 57 patients. Fourteen patients underwent second treatment, and one patient had third treatment. The overall survival rates were $72.1 \%$ at 3 years and $66.7 \%$ at 5 years for all patients and $62.4 \%$ at 3 years and $54.5 \%$ at 5 years for germ cell tumors (GCTs). The LTCRs were $94.30 \%$ at 3 years and $90.80 \%$ at 5 years for all patients and $88.00 \%$ at 3 years and $77.27 \%$ at 5 years for GCTs.
\end{abstract}

Conclusions: GKRS is an effective and safe modality that can be widely used to PRTs as the primary therapy.

Keywords: Gamma knife radiosurgery, Pineal region tumors, Germ cell tumors, Follow-up

\section{Background}

The pineal region tumors (PRTs) are a deep-seated, heterogeneous group of mass lesions, which are derived from cells located in or adjacent to the pineal gland. PRTs are rare, which accounts for $0.5-1 \%$ of all intracranial tumors in the United States and Europe [1] but have a higher incidence of $3.2 \%$ in Japan [2, 3], $0.9 \%$ in Islamabad [4], $1.2 \%$ in Karachi [5], and $1.9 \%$ in China [6]. These tumors are approximately ten times more common in children than in adults and constitute 3-8 \% of pediatric intracranial tumors [7].

Due to heterogeneous cellular origins, PRTs represent a spectrum of neoplasms ranging from benign (teratoma, meningioma, pineocytoma, ependymoma, etc.) to malignant (pineoblastoma, malignant teratoma, malignant germ cell tumors, etc.). The management strategies for these

\footnotetext{
* Correspondence: jinningsong@126.com

Wentao Li, Wenxing Kang, and Boning Dong are co-first authors.

${ }^{\dagger}$ Equal contributors

'Department of Neurosurgery, the First Affiliated Hospital, Medical School of Xi'an Jiaotong University, \#277, Yantaxi Rd, Xi'an 710061, China

Full list of author information is available at the end of the article
}

tumors remain controversial [8]. Some investigators state that surgical resection should be used as the initial therapeutic modality [9-11] and that histologic diagnosis of PRTs is required for rational treatment. In contrast, some Asian investigators [12] recommend a test dose of radiation therapy based on the high incidence of germinoma in their countries. There are regional differences in the incidence of GCTs in the pineal region. In Asian, germ cell tumors (GCTs) are more common overall (accounting for $3 / 1 \%$ of all primary brain tumors in Japan [13]/China, respectively [6]) but are predominantly the more benign germinomas. In Japan, GCTs account for $70.3 \%$ of pineal region tumors $[6,13]$. Some experts in Japan advocate empiric radiosurgery $[14,15]$ for reducing the cost of treatment and the need for hospitalization. Several clinical studies have demonstrated that gamma knife radiosurgery (GKRS) has high efficacy and low morbidity [16], and thus has the potential as a primary treatment modality $[17,18]$.

On the whole, GKRS is safe and effective for pineal region tumors. The most successful GKRS treatment of pineal region tumors has been with benign or low-grade 
lesions. The three broad categories of tumor treated by GKRS include pineal parenchymal tumors (pineocytomas and pineoblastomas), GCTs (germinomatous and nongerminomatous), and glial tumors (astrocytomas).Pure germinomas are exquisitely radiosensitive. Many studies [11, 18-20] mainly used GKRS as an adjuvant therapy rather than as a primary treatment modality. Only a few studies explore the efficacy of GKRS in treating pineal region tumors in series as a primary method, and the numbers of cases were small [19-21]. There is a need for larger studies with longer follow-up before GKRS becomes widely adopted in the treatment of pineal region tumors. In this report, we reviewed our institutional experiences of treating pineal region tumors by GKRS in 147 patients.

\section{Methods}

Ethics, consent, and permissions

This study was approved by the First Affiliated Hospital of Xi' an Jiaotong University Institutional Review Board and met the requirements of medical ethics. Consents to participate the study from the participants (or legal parent or guardian for children) were obtained.

\section{Consent to publish}

We had obtained consent to publish from the participants (or legal parent or guardian for children) to report individual patients' data in any form (including images, videos, voice recordings, etc.).

\section{Patient population and diagnosis}

We retrospectively reviewed 147 consecutive cases of pineal region tumors treated with GKRS at the First Affiliated Hospital of Xi'an Jiaotong University (Xi'an, China) between 1999 and 2009. All subjects were Han Chinese from Northwest China. Inclusion criterion is: all patients were newly diagnosed incident PRT cases undergoing GKRS at our institution. Exclusion criterion: patients with arteriovenous malformations and brain metastases.

Diagnosis was mainly based on magnetic resonance imaging (MRI; GE HDxt 3.0T, USA) combined with medical history, clinical presentations, Karnofsky performance score [22], and patient age. T1-weighted and T2-weighted MRI were performed. MRI scans were assessed independently by two radiologists blinded to the clinical outcome.

\section{GKRS technique}

Based on data from computer tomography (CT) and MRI, patients of progressive hydrocephalus underwent ventricular peritoneal (VP) shunt 7 days before GKRS (Elekta Instruments, Atlanta, GA, USA). The Leksell GammaPlan treatment planning software (versions 4.10-5.34, Elekta, Sweden) was used on all patients. Between 1 and 9 isocenters (mean 6.24 isocenters) were used in treatment planning. The Automated Positioning System (Elekta) was used to streamline patient positioning. Central dose was 25-40 Gy (mean \pm SD $36.34 \pm 2.11$ Gy), and marginal dose was $9-15$ Gy (mean \pm SD $13.6 \pm 1.02$ Gy).

\section{Patient assessment and follow-up}

The duration of follow-up ranged from 60.5 to 100.1 months (mean 67 months). The results of GKRS were mainly evaluated by changes in tumor size on MRI at $1,3,6$, and 12 months after GKRS. Meanwhile, the neurological presentations were evaluated. The systemic criteria proposed by the Japan Brain Tumor Registry [23] were used. This system is composed of five grades including complete response (CR; tumor disappearance), partial response (PR; $\geq 50 \%$ tumor volume reduction), minor response (MR; $25-50 \%$ reduction), no change (NC; $<25 \%$ reduction), and progression (tumor enlargement). The local tumor control rate was calculated as $(\mathrm{CR}+\mathrm{PR}+\mathrm{MR}+\mathrm{NC}) /$ total.

\section{Results}

\section{Patients' characteristics}

We identified 158 lesions in the 147 patients included in this study. Eight patients had multiple lesions. Most lesions were located in the pineal region. The demographics/clinical presentation of patients are summarized in Table 1 . There were 105 males and 42 females, with a ratio of 2.5:1. Patients' ages ranged from 6 to 74 years (mean \pm SD $35.6 \pm 12.1$ years). The mean volume of PRTs before GKRS treatment was $8.47 \mathrm{~cm}^{3}$ (SD $6.22 \mathrm{~cm}^{3}$ ). The duration between the onset of symptoms and admission at our institute ranged from 3 months to 2 years. The most

Table 1 Clinical presentations of patients with germ cell tumors (GCTs) and non-germ cell tumors (non-GCTs)

\begin{tabular}{lll}
\hline Classification & Non-GCTs & GCTs \\
\hline No. of cases & 107 & 40 \\
Age & $42.3 \pm 10.2$ & $14.4 \pm 6.1$ \\
$\quad 17$ & 6 \\
$\quad$ Minimum age & 74 & 20 \\
Meximum age & & \\
Male & 74 & 31 \\
Female & 33 & 9 \\
Clinical symptoms & & \\
Headache & 59 & 38 \\
Nausea and vomiting & 40 & 29 \\
Polydipsia and polyuria & 9 & 32 \\
Eye movement disorders & 23 & 18 \\
Parinaud sindromas & 16 & 19 \\
Precocious puberty & 2 & 1 \\
Hypogenesis & 1 & 4 \\
Ataxia & 15 & 12 \\
\hline
\end{tabular}


common symptoms were headache, nausea, and vomiting. Other symptoms included polydipsia, polyuria, ataxia, eye movement disorders, parinaud syndromes, precocious puberty, and hypogenesis.

\section{GKRS treatment}

Patients first underwent MRI assessment to locate brain tumors. The average Karnofsky score was 72.23. A total of 104 (66 \%) lesions occurred in adults and 54 (34\%) occurred in children. The lesions showed clear boundary. Some lesions had cystic changes, and gadodiamideenhanced (OmniScan, GE Healthcare) MRI showed uniform enhance mentor partial enhancement in cystic areas. Eight patients had multiple lesions located in the pineal region, parietal lobe, and occipital lobe. Forty patients were diagnosed as germ cell tumors. The mean tumor volume was $8.47 \mathrm{~cm}^{3}\left(0.1-29.2 \mathrm{~cm}^{3}\right)$, and the maximum tumor diameter was $3.8 \mathrm{~cm}$. Dosimetry of GKRS is listed in Table 2.

\section{Follow-up}

After GKRS treatment, three patients developed complications including severe headache, projectile vomiting and subsequent coma and were transferred to the intensive care unit. These patients were mainly given life-sustaining treatment and treatment to reduce intracranial pressure. One patient developed acute brain herniation due to rapidly progressing severe brain edema and died. The other two patients regained consciousness after their brain edema disappeared and were transferred to a general ward. They did not experience any other discomfort during the follow-up period after leaving the hospital. Fatigue was seen in 103 patients, headache in 97 patients, and nausea and vomiting in 69 patients. After reducing intracranial pressure using mannitol, these symptoms were alleviated and gradually disappeared.

At 2 months after GKRS, lesions in 91 cases (61.9\%) were significantly reduced, and symptoms were improved or disappeared in 121 cases (83.31\%). At 6 months, radiographic review showed that the average tumor volume was reduced to $4.2 \mathrm{~cm}^{3}$ (range $0-19.7 \mathrm{~cm}^{3}$ ) from $8.47 \mathrm{~cm}^{3}$ before GKRS. The pineal region tumor volume was reduced, and saddle or occipital lesions disappeared in 123 cases (83.67 \%). Tumors in 17 cases increased in size,

Table 2 Dosimetry of GKRS

\begin{tabular}{ll}
\hline Items & Mean $\pm \mathrm{SD}$ \\
\hline Tumor volume $\left(\mathrm{cm}^{3}\right)$ & $8.47 \pm 6.22$ \\
No. of isocenters & $6.24 \pm 1.11$ \\
Central dose (Gy) & $36.34 \pm 2.11$ \\
Marginal dose (Gy) & $13.6 \pm 1.02$ \\
Maximum dose (Gy) & $28.35 \pm 5.37$ \\
\hline
\end{tabular}

GKRS Gamma knife radiosurgery, SD standard deviation but tumor density was reduced in 14 of these cases. The average follow-up duration was 67.2 months (range 60.5100.1). The tumor disappeared in 57 patients after 1 year (Table 3). The overall survival rates for 1,3 , and 5 years after GKRS were 80.2, 72.1, and $66.7 \%$, respectively. Local tumor control rates were 97.40, 94.30, and $90.80 \%$ for 1,3 , and 5 years, respectively.

We also analyzed the GCT cases separately. In the GCT sub-group (40 cases), symptoms were improved in 13 cases and completely disappeared in 24 cases at 2 months post GKRS. At 6 months, lesions disappeared in eight cases according to MRI scans. Another 8 cases showed residual lesions and underwent second radiosurgery. There was no death for the 40 cases of GCTs. The 1-, 3-, and 5-year overall survival rates for GCTs (Table 4) were $77.5,62.4$, and $54.5 \%$, respectively. The local tumor control rates were 96.70, 88.00, and $77.27 \%$, for 1,3 , and 5 years, respectively.

Fourteen patients in this study (eight of GCTs and six of non-GCTs) underwent second treatment, and one of these patients had third treatment. Eight of these patients had multiple lesions that are either GCTs or had a total volume of $21.5-28.1 \mathrm{~cm}^{3}$. One patient had polyuria during the first 3 months after treatment. The patients' MRI revealed a new lesion in suprasellar region oppressing pituitary stalk. Since the volume of pineal region lesion did not reduce, this patient was given low dose of whole brain radiation therapy. Five months later, polyuria and the lesion in suprasellar region disappeared, and pineal region tumor shrank.

\section{Discussion}

In this study, we reviewed the results of GKRS in 147 patients. The average duration of follow-up was 67.2 months. The 3/5-year survival rates for patients after GKRS treatment were $72.1 / 66.7 \%$ for all patients and 62.4/ $54.5 \%$ for GCTs. The 3/5-year local tumor control rates were $94.30 / 90.80 \%$ for all patients and $88.00 / 77.27 \%$ for GCTs, respectively.

The results indicate that GKRS without histological verification of PRTs is acceptable. It has been controversial whether histological diagnosis before treatment is necessary $[10,18,24]$ in the management of PRTs, partly owing to racial differences in incidence $[1-3,6]$. Therefore, it is not clear which treatment options are most beneficial.

With the advances of clinical image (MRI) and clinical features, we can now diagnose PRTs and differentiate GCTs from non-GCTs without histological analysis. Therefore, GKRS can be performed either as a primary therapy or as an adjuvant therapy for conventional treatments. Kanamori [24] reported that the histological diagnoses of 38 out of 39 GCT patients and all of eight non-GCT patients were similar to their clinical diagnoses. Konovalov reported that [10] surgery or GKRS for 
Table 3 Evaluation of all (147) cases after GKRS treatment

\begin{tabular}{lllllllllll}
\hline & CR (\%) & PR (\%) & MR (\%) & NC (\%) & PROGR (\%) & Total & LTCR (\%) & ATV $\left(\mathrm{cm}^{3}\right)$ & Karnofsky scoring & Survival rate (\%) \\
\hline Before treatment & & & & & & & & 8.47 & 72.23 \\
1 year after treatment & $57(48.31)$ & $34(28.81)$ & $22(18.64)$ & $2(1.69)$ & $3(2.54)$ & 118 & 97.40 & 1.23 & $87.83 \pm 7.31$ & 80.2 \\
3 years after treatment & $52(49.06)$ & $27(25.41)$ & $19(17.92)$ & $2(1.89)$ & $6(5.66)$ & 106 & 94.30 & 1.33 & $77.25 \pm 13.38$ & 72.1 \\
5 years after treatment & $43(43.88)$ & $26(26.53)$ & $17(17.35)$ & $3(3.06)$ & $9(9.18)$ & 98 & 90.80 & 2.29 & $76.43 \pm 17.76$ & 66.7 \\
\hline
\end{tabular}

Abbreviations: GKRS Gamma knife radiosurgery, $C R$ complete response (tumor disappearance), $P R$ partial response ( $\geq 50 \%$ tumor volume reduction), $M R$ minor response ( $25-50 \%$ reduction), $N C$ no change ( $<25 \%$ reduction), PROGR progression (tumor enlargement), $L T C R$ local tumor control rate $=C R+P R+M R+N C /$ total, ATV average tumor volume

patients with butterfly-shaped radiologic sign in MRI were successful without pathologic confirmation of diagnosis. Our institutional experiences support this option.

In our study, the 5 -year survival rate for patients with PRTs is $66.7 \%$; however, the survival rate in Kanamori's study [24] was $94 \%$ without histological verification. The difference in survival rate could be due in part to the fact that there was no neoadjuvant chemotherapy in our study. Alternatively, it could be due to differences in the number of cases (147 cases in our study and 39 cases in Kanamori's) or patient population. The 5-year local tumor control rate in our study was $90.80 \%$, which is higher than $70 \%$ in an earlier study of 49 cases [20]. In the present study, the 1-, 3-, and 5-year overall survival rates were $77.5,62.4$, and $54.5 \%$, respectively, in GCTs cases (40 cases) after GKRS. Mori reported that [20] survival rates at 5 and 10 years after GKRS in GCT cases $(n=38)$ were both $68 \%$. The difference in survival rate could be due in part to diagnosis. In 13 of 38 patients was histologically diagnosed, but in others GCT was diagnosed by elevation of serum tumor markers or clinical course. In our study, GCT was diagnosed by MRI, medical history, and clinical presentations.

A definitive histological verification is surely desirable, but successful treatment methods without the need for biopsy have been developed [24, 25]. It is interesting that the survival rates are not significantly influenced by the unclear histological diagnosis. This phenomenon likely results from the large variety of tumor types in the pineal region running necessary biopsies in some circumstances.

With the development of neurosurgery and the emergence of CT, MRI, and surgical microscope, it is possible to achieve complete removal and secondary complete removal. Therefore, there has been a significant decrease in death rate. Microsurgical mortality is under $8 \%$ and disability rate is under $12 \%$ [26]. Radiotherapy is safe and effective, which makes it a good alternative treatment, especially for germ cell tumors that are sensitive to radiation. There are still disagreements over the treatment of pinealoma. Considering the efficacy, imbalance of domestic economic development, as well as cost-effectiveness of surgical total resection and subtotal resection in developing countries [27], it is difficult to achieve a wide range of application for surgical total resection and subtotal resection. As such, radiosurgery cannot be replaced by surgery because it is a non-time-consuming, economic, and less invasive therapy.

GKRS can not only kill the tumor cells but also reduce the damage to the surrounding normal brain tissues [28], thanks to its technical characteristic of large dose in targeted area and low dose on the edge [29]. Therefore, GKRS is associated with less systemic side effects compared to conventional radiation. Moreover, it can also be used to treat tumors that are insensitive to conventional radiotherapy.

Pinealoma is usually accompanied by obstructive hydrocephalus. Sixteen patients $(10.9 \%)$ in this study had Parinaud's syndrome, which is a sign of acute obstructive hydrocephalus. In order to prevent perioperative and postoperative intracranial hypertension, VP shunt was performed on patients with progressive hydrocephalus. In the present study, patients treated with mannitol after surgery had no intracranial hypertension, infection, or shunt malfunction. These findings indicate that we can improve the safety of GKRS by actively dealing with hydrocephalus.

Given that pinealoma may metastasize by seeding through the cerebrospinal fluid pathways, there is a

Table 4 Evaluation of GCTs (40 cases) after GKRS treatment

\begin{tabular}{|c|c|c|c|c|c|c|c|c|c|c|}
\hline & CR (\%) & PR (\%) & MR (\%) & NC (\%) & PROGR (\%) & Total number & LTCR (\%) & $\operatorname{ATV}\left(\mathrm{cm}^{3}\right)$ & Karnofsky scoring & Survival rate (\%) \\
\hline Before treatment & & & & & & & & 7.28 & 76.25 & \\
\hline 1 year after treatment & $14(45.16)$ & $12(38.71)$ & $2(6.45)$ & $2(6.45)$ & $1(3.23)$ & 31 & 96.70 & 2.21 & $85.22 \pm 9.15$ & 77.5 \\
\hline 3 years after treatment & $11(44.00)$ & $8(32.00)$ & $1(4.00)$ & $2(8.00)$ & $3(12.00)$ & 25 & 88.00 & 2.54 & $72.52 \pm 14.58$ & 62.4 \\
\hline 5 years after treatment & $8(36.36)$ & $7(31.82)$ & $1(4.55)$ & $1(4.55)$ & $5(22.73)$ & 22 & 77.27 & 2.91 & $66.14 \pm 16.86$ & 54.5 \\
\hline
\end{tabular}

Abbreviations: GCTs germ cell tumors, GKRS gamma knife radiosurgery, $C R$ complete response (tumor disappearance), $P R$ partial response ( $\geq 50 \%$ tumor volume reduction), $M R$ minor response ( $25-50 \%$ reduction), $N C$ no change ( $<25 \%$ reduction), PROGR progression (tumor enlargement), LTCR local tumor control rate $=\mathrm{CR}+\mathrm{PR}+\mathrm{MR}+\mathrm{NC} /$ total, $A T V$ average tumor volume 
debate about whether radiotherapy or chemotherapy should be used. The rate of metastases from pinealoma is continually rising based on recent reports. Ogawa et al. [30] reviewed 126 cases of intracranial germ cell tumors that underwent full central radiotherapy and found that the incidence of spinal relapses was $4 \%$ (2 of 56) for patients treated with spinal irradiation and $3 \%$ ( 2 of 70 ) for those without spinal irradiation. Nguyen et al. [31] reviewed 21 patients with intracranial germinoma treated with radiotherapy and found that irradiation with chemotherapy is associated with increased rates of failures in the brain and spine.

\section{Conclusion}

In conclusion, our institutional experiences support that GCTs may be diagnosed based on MRI and clinical presentations without biopsy or surgery. Moreover, GKRS appears to be an effective, low-risk treatment option that can be widely used for pineal region tumors either as a primary therapy without histological diagnosis. Further studies are needed to evaluate the effectiveness of GKRS for the treatment of PRTs as a primary therapy in more details.

\section{Abbreviations}

CR: Complete response; CT: Computer tomography; GCTs: Germ cell tumors; GKRS: Gamma knife radiosurgery; LTCRs: Local tumor control rates; MR: Minor response; NC: No change; PR: Partial response; PRTs: Pineal region tumors; VP: Ventricular peritoneal.

\section{Competing interests}

The authors declare that they have no competing interests.

\section{Authors' contributions}

WL, BZ, and WK drafted the manuscript and provided clinical data. BD and XM collected case information, read manuscript, and performed the statistical analysis. JS participated in the design of the study and reviewed manuscript. YL and ZL carried out technical support and performed the statistical analysis. All authors read and approved the final manuscript.

\section{Acknowledgements}

This study was supported by the Special Research Fund for the Doctoral Disciplinary Points in Universities of Ministry of Education (No. 20110201110060).

\footnotetext{
Author details

'Department of Neurosurgery, the First Affiliated Hospital, Medical School of Xi'an Jiaotong University, \#277, Yantaxi Rd, Xi'an 710061, China. ${ }^{2}$ Honghui Hospital, Xi'an Jiaotong University Health Science Center, Xi'an 710054, China. ${ }^{3}$ Department of Radiation Oncology, 323 Hospital of Chinese People's Liberation Army, Xi'an 710000, China. ${ }^{4}$ Beilin Kangjie Hospital, Xi'an 710000, China. ${ }^{5}$ Department of Neurosurgery, the Northwest Civil Aviation Hospital, Xi'an 710061, China. ${ }^{6}$ Department of Neurosurgery, the Dingxi First People's Hospital, Dingxi 743000, China.
}

Received: 22 March 2015 Accepted: 12 October 2015

Published online: 21 October 2015

\section{References}

1. Cushing H. Intracranial tumours: notes upon a series of two thousand verified cases with surgical-mortality percentages pertaining thereto. Springfield: Charles C. Thomas; 1932.

2. Apuzzo MLJ. Surgery of the third ventricle. Baltimore: Williams \& Wilkins; 1998.
3. Zulch KJ. Brain tumors: their biology and pathology. Berlin: Springer-Verlag; 1986.

4. Nasir S, Jamila B, Khaleeq S. A retrospective study of primary brain tumors in children under 14 years of age at PIMS, Islamabad. Asian Pac J Cancer Prev. 2010;11:1225-7.

5. Ahmed N, Bhurgri Y, Sadiq S, Shakoor KA. Pediatric brain tumours at a tertiary care hospital in Karachi. Asian Pac J Cancer Prev. 2007;8:399-404.

6. Zang X. Pathological analysis of 1344 cases of intracranial tumors. Zhonghua Shen Jing Jing Shen Ke Za Zhi. 1982;15:228-33.

7. Abay 2nd EO, Laws Jr ER, Grado GL, Bruckman JE, Forbes GS, Gomez MR, et al. Pineal tumors in children and adolescents. Treatment by CSF shunting and radiotherapy. J Neurosurg. 1981;55:889-95.

8. Yianni J, Rowe J, Khandanpour N, Nagy G, Hoggard N, Radatz M, et al. Stereotactic radiosurgery for pineal tumours. Br J Neurosurg. 2012;26:361-6.

9. Jamieson KG. Excision of pineal tumors. J Neurosurg. 1971;35:550-3.

10. Konovalov AN, Pitskhelauri DI. Principles of treatment of the pineal region tumors. Surg Neurol. 2003;59:250-68.

11. Schild SE, Scheithauer BW, Haddock MG, Wong WW, Lyons MK, Marks LB, et al. Histologically confirmed pineal tumors and other germ cell tumors of the brain. Cancer. 1996;78:2564-71.

12. Blakeley JO, Grossman SA. Management of pineal region tumors. Curr Treat Options Oncol. 2006;7:505-16.

13. Nomura K. Epidemiology of germ cell tumors in Asia of pineal region tumor. J Neurooncol. 2001;54:211-7.

14. Choi JU, Kim DS, Chung SS, Kim TS. Treatment of germ cell tumors in the pineal region. Childs Nerv Syst. 1998;14:41-8.

15. Endo $H$, Kumabe $T$, Jokura $H$, Tominaga T. Stereotactic radiosurgery followed by whole ventricular irradiation for primary intracranial germinoma of the pineal region. Minim Invasive Neurosurg. 2005;48:186-90.

16. Hyun MK, Hwang JS, Kim JH, Choi JE, Jung SY, Bae JM. Survival outcomes after whole brain radiation therapy and/or stereotactic radiosurgery for cancer patients with metastatic brain tumors in Korea: a systematic review. Asian Pac J Cancer Prev. 2013;14:7401-7.

17. Reyns N, Hayashi M, Chinot O, Manera L, Peragut JC, Blond S, et al. The role of gamma knife radiosurgery in the treatment of pineal parenchymal tumours. Acta Neurochir (Wien). 2006;148:5-11. discussion 11.

18. Hanft SJ, Isaacson SR, Bruce JN. Stereotactic radiosurgery for pineal region tumors. Neurosurg Clin N Am. 2011;22:413-20. ix.

19. Kano H, Niranjan A, Kondziolka D, Flickinger JC, Lunsford D. Role of stereotactic radiosurgery in the management of pineal parenchymal tumors. Prog Neurol Surg. 2009;23:44-58.

20. Mori Y, Kobayashi T, Hasegawa T, Yoshida K, Kida Y. Stereotactic radiosurgery for pineal and related tumors. Prog Neurol Surg. 2009;23:106-18,

21. Lekovic GP, Gonzalez LF, Shetter AG, Porter RW, Smith KA, Brachman D, et al. Role of gamma knife surgery in the management of pineal region tumors. Neurosurg Focus. 2007;23:E12.

22. Karnofsky DA, Abelmann WH, Craver LF, Burchenal JH. The use of the nitrogen mustards in the palliative treatment of carcinoma. With particular reference to bronchogenic carcinoma. Cancer. 1948;1:634-56.

23. Japan CoBTRo. The criteria of the effects of treatments for brain tumors. Tokyo: Kanehara Publisher; 1995.

24. Kanamori M, Kumabe T, Tominaga T. Is histological diagnosis necessary to start treatment for germ cell tumours in the pineal region? I Clin Neurosci. 2008;15:978-87.

25. Chasan CB, Goetsch S, Ott K. Radiosurgery for pineal tumors: is biopsy indicated? Stereotact Funct Neurosurg. 1996;66 Suppl 1:157-63.

26. Bruce JN, Ogden AT. Surgical strategies for treating patients with pineal region tumors. J Neurooncol. 2004;69:221-36.

27. Rabadan AT, Hernandez D, Eleta M, Pietrani M, Baccanelli M, Christiansen $S$, et al. Factors related to surgical complications and their impact on the functional status in 236 open surgeries for malignant tumors in a Latino-American hospital. Surg Neurol. 2007;68:412-20. discussion 420.

28. Duan L, Zeng R, Yang KH, Tian JH, Wu XL, Dai Q, et al. Whole brain radiotherapy combined with stereotactic radiotherapy versus stereotactic radiotherapy alone for brain metastases: a meta-analysis. Asian Pac J Cancer Prev. 2014;15:911-5.

29. Hasegawa T, Kondziolka D, Hadjipanayis CG, Flickinger JC, Lunsford LD. The role of radiosurgery for the treatment of pineal parenchymal tumors. Neurosurgery. 2002;51:880-9. 
30. Ogawa K, Shikama N, Toita T, Nakamura K, Uno T, Onishi H, et al. Long-term results of radiotherapy for intracranial germinoma: a multi-institutional retrospective review of 126 patients. Int J Radiat Oncol Biol Phys. 2004;58:705-13.

31. Nguyen QN, Chang EL, Allen PK, Maor MH, Ater JL, Mahajan A, et al. Focal and craniospinal irradiation for patients with intracranial germinoma and patterns of failure. Cancer. 2006;107:2228-36.

Submit your next manuscript to BioMed Central and take full advantage of:

- Convenient online submission

- Thorough peer review

- No space constraints or color figure charges

- Immediate publication on acceptance

- Inclusion in PubMed, CAS, Scopus and Google Scholar

- Research which is freely available for redistribution 